\title{
Endometrial vessel maturation in women exposed to levonorgestrel- releasing intrauterine system for a short or prolonged period of time
}

Ravet Stéphanie ${ }^{1, \dagger}$ Soraya Labied ${ }^{1, \dagger}$, Silvia Blacher ${ }^{1}$, Francis Frankenne ${ }^{1}$, Carine Munaut ${ }^{1}$, Viviana Fridman ${ }^{2}$, Aude Beliard ${ }^{1}$, Jean-Michel Foidart ${ }^{1,3, \dagger}$ and Michelle Nisolle ${ }^{1,3, \uparrow}$

${ }^{1}$ Laboratory of Tumor and Development Biology, Center of Experimental Cancer Research (CECR), University de Liège, Tour de Pathologie (B23), Avenue de l'hôpital, GIGA-R, Sart-Tilman, B-4000 Liège, Belgium; ${ }^{2}$ Department of Anatomopathology, Citadelle, University of Liège, B-4000 Liège, Belgium; ${ }^{3}$ Department of Gynecology, CHU, University of Liège, B-4000 Liège, Belgium

${ }^{\dagger}$ These authors contributed equally.

\begin{abstract}
:
BACKGROUND: Levonorgestrel-releasing intrauterine system (LNG-IUS), although inserted to reduce heavy menstruation, causes irregular early transient bleeding. The objective of the study was to document quantitative changes in endometrial vessels of short- ( $\leq 3$ months) and long-term ( $\geq 12$ months) LNG users. The area, density and maturation of endometrial vessels were quantified in 19 endometrial biopsies of women with LNG-IUS and in 10 normally ovulating patients during mid-luteal phase.

METHODS: Vessel maturation was evaluated by double immunostaining using anti-von Willebrand factor (endothelial cell marker) and anti-alpha Smooth Muscle Actin (vascular smooth muscle cells) antibodies. Vessel area, number and density were quantified with a novel computer-assisted image analysis system.

RESULTS: Endometrium exposed to LNG-IUS for 1-3 months displayed a 11.5-fold increase in small naked vessel number. The partially mature vessel ( $\alpha$ SMA partially positive) number increased six times. After longterm LNG-IUS treatment, the immature and partially mature vessel number remained four times higher than in the control group. Vessel area and density also increased dramatically in a time-dependent pattern with LNGIUS use.

CONCLUSIONS: Levonorgestrel affects blood vessel number, area, density and maturation in a time-dependent pattern that may explain the early transient increase in breakthrough bleeding with the LNG-IUS.
\end{abstract}

Keywords: endometrium; levonorgestrel; bleeding; vascularization; image analysis

\section{INTRODUCTION}

Long-term progestin-only contraceptive methods such as the levonorgestrel-releasing intrauterine system (LNGIUS) lead to irregular and abnormal endometrial spotting and bleeding. This unpredictable breakthrough bleeding (BTB), which causes considerable social discomfort and reduces quality of life, is the most common reason for discontinuation of use in $\sim 25 \%$ of women (Vekemans, 1995; Vekemans et al, 1997). It occurs predominantly during the first few months of treatment and then progressively and spontaneously decreases.

The exact mechanism responsible for these bleedings is unknown and the aetiology seems to be multifactorial. Several studies have demonstrated a possible contribution of matrix metalloproteinases (MMPs) (Skinner et al., 1999; Vincent et al., 1999; Galant et al., 2000; Vincent et al., 2002; Chegini et al., 2003; Galant et al., 2004), tissue factor (TF) (Runic et al., 2000) and granulocyte-macrophage cytokines (Critchley et al., 1998). Anomalies in progesterone and estrogen receptor expression have been also shown to play a role in BTB associated with LNG-IUS (Critchley et al., 1998; Zhu et al., 1999).

Modifications in endometrial microvascular density and a decrease in vessel maturation due to a lack of surrounding pericytes and smooth muscle cells may also be involved in this process along with modifications of endothelial basement membrane structure and composition (Rogers et al., 1993; Hickey et al., 1999; Jondet et al., 2005). Following Norplant exposure, a reduction of basement membrane components around the endometrial endothelium has been demonstrated but without any correlation with bleeding episodes (Hickey et al., 1999).

In order to determine the extent of vascular remodelling in endometrium of women exposed to LNG-IUS, we compared, in this study, the area, density and maturation of blood vessels after short- or long-term exposure to LNG-IUS. 


\section{MATERIALS AND METHODS}

\section{Patients}

The study was approved by the Ethical Committee of the University Hospital. Nineteen endometrial biopsies were analysed. Eight biopsies (Group I) were obtained from women exposed to LNG-IUS (Mirena ${ }^{\circledR}$, Bayer Schering Pharma, Berlin, Germany) for a period of 1-3 months. In fact, these eight women (mean age 39 years old), with an indication of hysterectomy for benign gynaecological indication, were prospectively recruited. Written informed consent was obtained from each woman and LNG-IUS was inserted into each subject for a median period of 1 month prior to surgery. Eleven biopsies (Group II) were obtained from women with breakthrough bleeding (mean duration of exposure 36 months, range 12-60 months) who required LNG-IUS removal for pregnancy desire or LNG-IUS change. Biopsies were retrieved from the Department of Anatomopathology, University Hospital, Liège, Belgium.

The control group consisted of 10 endometrial biopsies, performed during the mid-secretory phase of a spontaneous documented ovulatory cycle of women with documented fertility. The ages of the women in each group are shown in Table 1. All women in both LNG-IUS groups had presented at least one episode of transient bleeding during the 2 weeks prior to biopsy, whereas no breakthrough bleeding was observed in women with ovulatory cycles.

Table I: Patients' distribution in short-term LNG, long-term LNG and control groups.

\begin{tabular}{cccc}
\hline & Control group & \multicolumn{2}{c}{ LNG-IUS group } \\
& & $\begin{array}{c}\text { Short-term LNG } \\
(1-3 \text { months })\end{array}$ & $\begin{array}{c}\text { Long-term LNG } \\
(12-60 \text { months })\end{array}$ \\
\hline Patients, $n$ & 10 & 8 & 11 \\
Age, Mean \pm SD & $33.1 \pm 3.1$ & $39.2 \pm 5.7$ & $41.7 \pm 7.4$ \\
\hline
\end{tabular}

\section{Endometrial tissues}

All biopsies were obtained with a Cornier Pipelle suction curette (C.C.D. International, Paris, France) that allows sampling of the functional layer of the endometrium. Tissue were fixed in $4 \%$ formaldehyde solution in phosphate buffer saline (PBS) and embedded in paraffin. Specimens obtained during the mid-secretory phase were classified according to classical criteria (Noyes et al., 1950).

\section{Von Willebrand factor/ $\alpha$-smooth muscle actin double immunostaining}

Serial sections $(5 \mu \mathrm{m})$ were mounted on silanized slides and used for immunochemistry. The endometrial vasculature was visualized by double immunostaining of endothelial cells and perivascular cells. The primary antibodies (Abs) used were a rabbit polyclonal anti-human von Willebrand factor (vWF, A082, Dako, Denmark) and a mouse monoclonal anti-alpha smooth muscle actin ( $\alpha$ SMA, clone 1A4, A2547, Sigma-aldrich, USA). Slides were washed in Tris-HC1, $\mathrm{pH} 7.6$ between all steps unless otherwise stated. Endogenous peroxidase was blocked with $3 \% \mathrm{H}_{2} \mathrm{O}_{2}$ for $20 \mathrm{~min}$ at room temperature (RT). Slides were incubated with normal sheep serum (NSS) (Hormonology laboratory, Marloie, Belgium) for $30 \mathrm{~min}$ at RT, directly followed by vWF Ab (1/500 in NSS/Tris $10 \%$ ) overnight at $4{ }^{\circ} \mathrm{C}$ A swine anti-rabbit immunoglobulin conjugated to peroxidase (P0217, Dako, Denmark) was used as a secondary Ab. DAB + (Liquid DAB+ substrate chromogen system, K3468, Dako, Denmark) was applied for $15 \mathrm{~min}$ at RT in the dark as a chromogen and sections were rinsed in $\mathrm{H}_{2} \mathrm{O}$. Slides were incubated with normal goat serum (NGS) (Hormonology laboratory, Marloie, Belgium) before being incubated with the second primary Ab, $\alpha \mathrm{SMA}\left(1 / 400\right.$ in NGS/Tris $10 \%$ ) for $90 \mathrm{~min}$ at $37^{\circ} \mathrm{C}$. A goat anti-mouse $\mathrm{Ab}$ conjugated to biotin (E 0433, Dako, Denmark) diluted 1/400 in Tris buffer for $30 \mathrm{~min}$ at RT was used as a secondary $\mathrm{Ab}$, followed by an incubation for $30 \mathrm{~min}$ at RT with streptavidine-alkaline phosphatase (D 0396, Dako, Denmark) diluted 1/500. Finally, Fast Red chromogen system (K4016, Dako, Denmark) was applied for 10 min at RT in the dark. Sections were rinsed in $\mathrm{H}_{2} \mathrm{O}$ and mounted in Aqua Polymount (Polysciences, Inc., Warrington, PA, USA). Negative controls were performed by replacing each primary Ab with normal serum.

\section{Myosin heavy chain staining}

Serial sections were stained either with $\alpha$ SMA or myosin heavy chain (MHC) Abs in order to compare the relative distribution, and to quantify the proportion, of $\alpha$ SMA positive vessel sections that also reacted with the anti-MHC Ab. 
The MHC was visualized by the following steps. First, slides were incubated with proteinase $\mathrm{K}(50 \mu \mathrm{g} / \mathrm{ml})$ diluted in Tris- $\mathrm{HCl}$ for $10 \mathrm{~min}$ at $37^{\circ} \mathrm{C}$, and then slides were heated in a microwave oven set at 1.4 bar and $126^{\circ} \mathrm{C}$ for $11 \mathrm{~min}$ in target retrieval buffer (M3558, DAKO, Denmark). Endogenous peroxidase was blocked in $3 \% \mathrm{H}_{2} \mathrm{O}_{2}$ for 20 min at RT, and an incubation with mouse anti-human MHC Ab (M3558, DAKO, Denmark) diluted 1/100 was conducted 60 min at RT. Sections were washed in PBS ( $5 \times 5$ min) before incubation with the appropriate secondary Ab (goat anti-mouse, P0217, DAKO, Denmark) conjugated to biotin diluted 1/400, for 30 min, at RT. Finally, sections were incubated for $30 \mathrm{~min}$ with Streptavidine conjugated to HRP, diluted 1/500 for 30 min at RT and staining was revealed with the DAB system.

\section{Staining of the argyrophilic fibrillar network}

Silver impregnation of histological sections was performed as previously described (Gordon and Sweets, 1936).

\section{Image analysis and measurement}

Slides were observed with an Olympus microscope (Omnilabo, Aartselaar, Belgium) and the entire section [mean area $78.73 \mathrm{~mm}$ (range 22.90-163.07 $\mathrm{mm}^{2}$ )] was analysed at magnification of $\mathrm{x} 400$ by two observators (S.L. and S.R.). As described by Rogers et al. (2000), a score (0, 1 or 2) was attributed to blood vessels according to the intensity of $\alpha$ SMA staining. A score of 0 represents absence of $\alpha \mathrm{SMA}$, score 1 represents irregular $\alpha$ SMA distribution around part of the vessel circumference and score 2 indicates a positive continuous staining of the entire vessel circumference (Fig. 1). The entire endometrial tissue sections were analysed by counting vessels in 20-100 consecutive fields covering the entire endometrial area.

The contour of each vessel type was drawn manually at $\mathrm{x} 400$ magnification using Photoshop software by three different colours: turquoise for score 0 , blue for score 1, red for score 2 and green for tissue boundaries (Fig. 2A). Then, the colour image was decomposed into its three components: red (R), green (G) and blue (B) (Russ, $1999 \mathrm{a}$ and $\mathrm{b}$ ). For example, in R image, components corresponding to vessels scored 2 were strongly contrasted. Each of the R, G and B image component was therefore processed independently in order to obtain a binary image in which pixels belonging to vessels were attributed a value 1 (white) and pixels belonging to the background were attributed a null value (black). Figure 2B-D shows binary images of vessels scored 0, 1 and 2, respectively.

For each binary image, the following parameters were determined: number of vessel sections, vessel $\operatorname{area}\left(\mu \mathrm{m}^{2}\right)$ and tissue area $\left(\mu \mathrm{m}^{2}\right)$. Image processing and measurements were performed with the software Aphelion 3.2 from Adsis (France) on a PC.

Figure 1: Illustration of aSMA immunostaining scoring. $\alpha$ SMA positive cells were labelled in red and endothelial cells were stained in brown with an anti-vWF Ab. (A) Score 0 vessels are $\alpha S M A$ negative, (B) score 1 vessels display discontinuous $\alpha S M A$ staining and $(C)$ score 2 vessels have continuous $\alpha$ SMA staining. Original magnification $x 400$
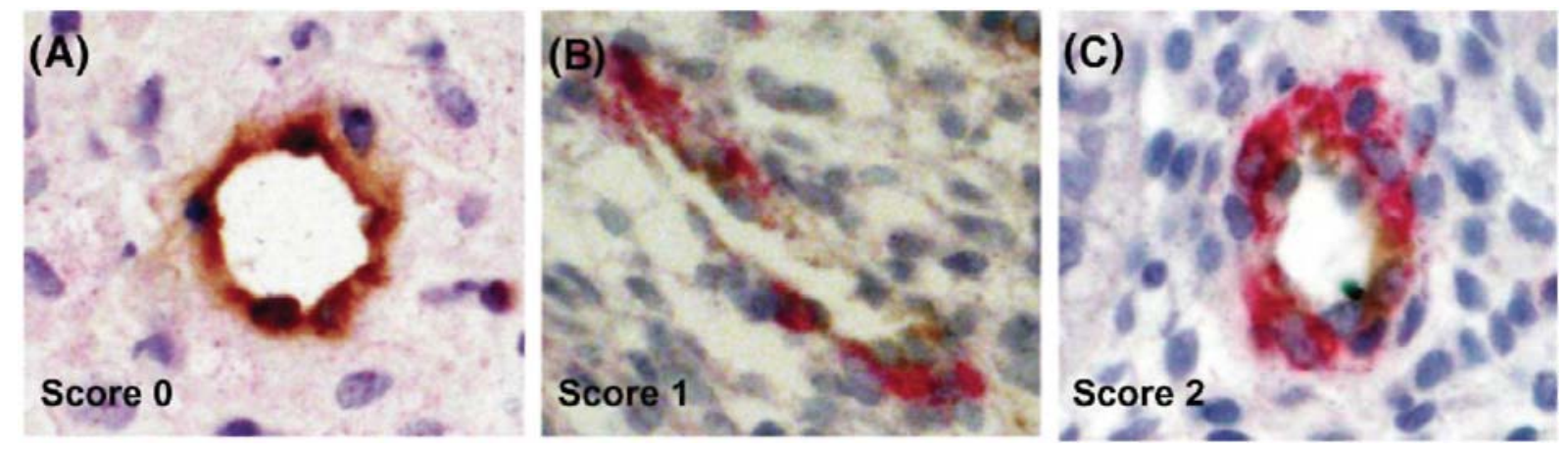
Figure 2: Image analysis and vessel quantification. (A) The contour of each type of vessel was drawn manually in the original image by the following different colours: turquoise for vessels scored 0, blue for vessels scored 1 and red for vessels scored 2. Green delineates the area of tissue in which measurements are performed. (B) Binary images of score 0 vessels, $(C)$ binary image of score 1 vessels and $(D)$ binary image of score 2 vessels contained in the original image
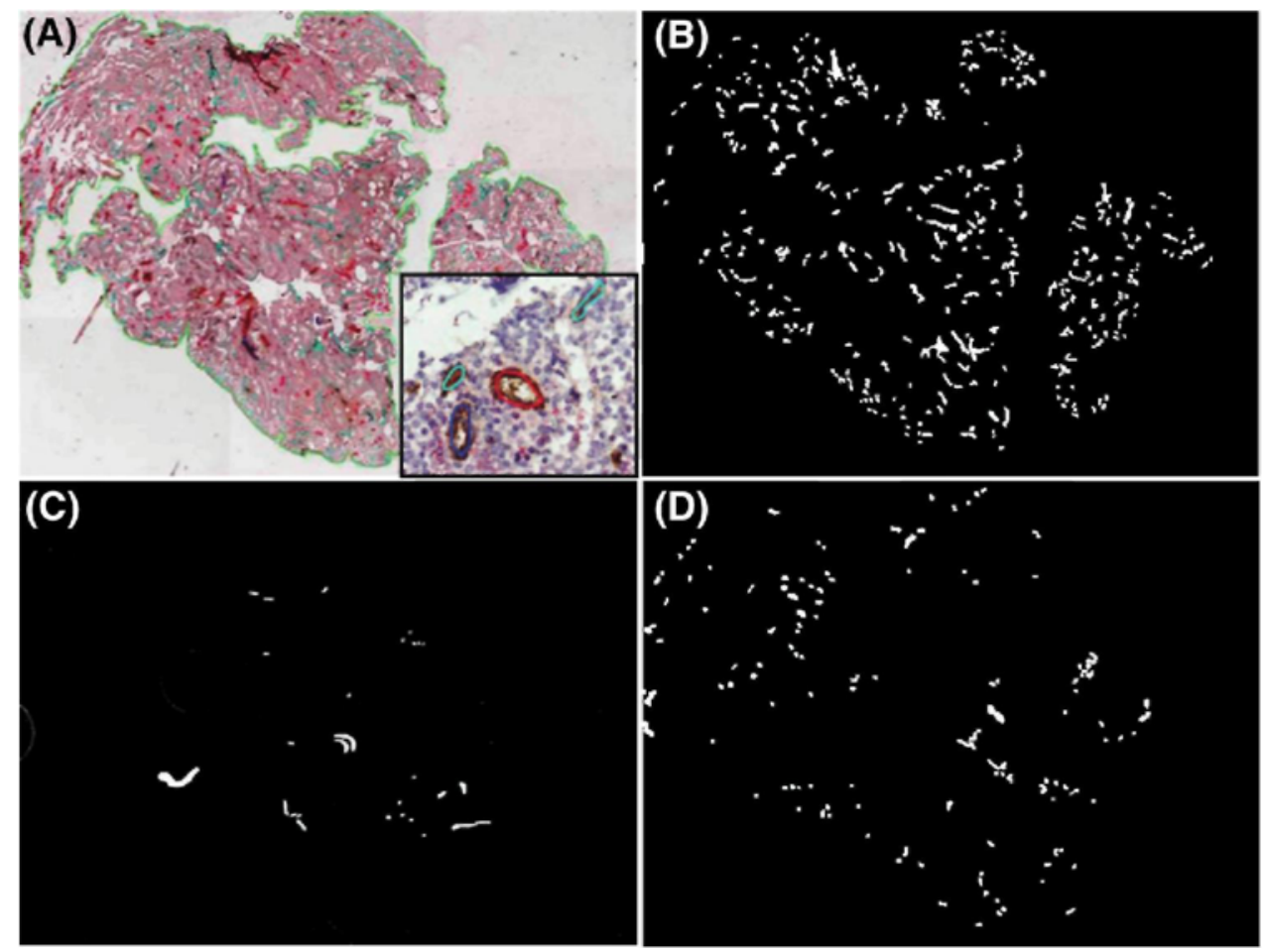

\section{Statistic analysis}

The number and area of vessels, as well as the corresponding area of tissue measured on each image, were analysed in the following two different ways: (i) vessel number per unit of tissue area $\left(\mathrm{n} / \mathrm{mm}^{2}\right)$ and relative vascular area defined as the proportion of tissue area occupied by the vessels (\%) were calculated for each image individually. Then, the mean value of these parameters was determined for each patient. Finally, the mean \pm SEM vessel number per unit of tissue area and the relative vascular area for all patients of the same group were determined, (ii) The vessel number per unit of tissue area and the relative vascular area were calculated for each patient. To do this, the number (or the area) of vessels measured on all five images of a patient were added and divided by the sum of the area of the tissue in the same five images. This gives only one value of the vessel number per unit of tissue area and of the relative vascular area by patient. Finally, the mean \pm SEM of those parameters was calculated for all the patients of the same group. As the area of the tissue considered on each image was not the same, the two calculations do not coincide exactly but follow closely the same trends, which suggest that the spatial distribution of vessels is rather homogeneous. Then, for the sake of simplicity, only the results obtained with the first method are discussed in the present paper. Relative vascular area and relative vessel number were represented as a percentage for each vessel category, calculated from values determined according to the method (i).

Analyses for statistical significance were evaluated with non-parametric methods. Kruskal-Wallis test with Dunn's correction for multiple comparisons was first applied. Then, for the cases in which significant differences were found between two groups, Mann-Whitney U-test was used to confirm this difference. Statistical significance was set at $P<0.05$.

\section{RESULTS}

\section{Stromal breakdown and fiber lysis}

Stromal breakdown occurring in women with endometrial breakthrough bleeding (BTB) (Galant et al., 2000; Kokorine et al., 1996) was documented by staining of argyrophilic fibrillar network using silver impregnation. 
Focal lysis of the collagen-rich fibrillar network was observed in all biopsies obtained after LNG-IUS use in the short- and long-term LNG groups but in none of those obtained from the control group (Fig. 3). This was consistent with the mild bleeding reported by women incorporated in the study. The area of stromal breakdown represented $<5 \%$ of total tissue area. The mean number of vessel sections $/ \mathrm{mm}$ in such areas was reduced by 40 $70 \%$ (data not shown). Therefore all subsequent evaluation of vessel characteristics was performed in intact zones.

\section{Vessel characterisation}

Three types of endometrial vessels were identified by double vWF- $\alpha$ SMA immunostaining. (i) Vascular endothelial cells that react with the anti-human vWF were the only cell types in some vessels (Fig. 1A-Score 0). (ii) Vessels coated with a discontinuous layer of pericytes identified by their $\alpha$ SMA content (Fig. 1B-Score 1). (iii) A continuous layer of $\alpha$ SMA positive cells surrounded by another fraction of vessel sections (Fig. 1CScore 2).

In addition, we compared distribution of $\alpha \mathrm{SMA}$ and $\mathrm{MHC}$ in vascular pericytes, since the former is a marker of smooth muscle cell differentiation, although the presence of myosin correlates with a functional contractile competence of vessels (Rogers and Abberton, 2003; Abberton et al., 1999). Myosin distribution closely correlates with $\alpha$ SMA pattern in endometrial vessels of the three groups (Fig. 4A). Quantitative evaluation indicated that $\sim 70 \%$ of vessels coated with pericytes ( $\alpha$ SMA positive) also contained myosin and were contractile (Fig. 4B). This percentage was identical among the three groups of women.

Figure 3: Focal stromal breakdown and lysis of the collagen-rich argyrophilic fibrillar network observed using silver impregnation. Control group (A) short-term LNG group (delimitated zone) (B) and long-term LNG group (delimitated zone) (C) Original magnification $\times 100$
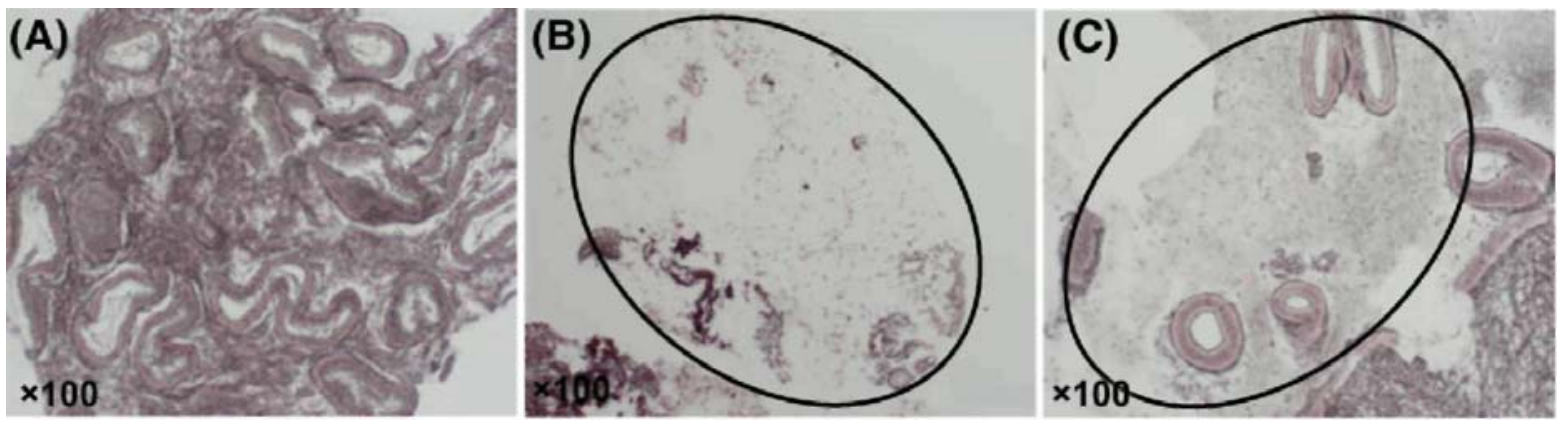

\section{The number of vessel sections is increased in endometrium of LNG-IUS users}

The number of vessel sections per mm was counted by a novel computer assisted method from binary structure after image transformation. In total, 11093 vessel sections were counted for the three groups (Fig. 5). A statistically significant increase in vessel section number of immature $(11.5 \mathrm{x})(P=0.0002)$ and partially mature (6x) $(P=0.0018)$ vessels was observed in short-term treated patients (Group I) compared with control group. Less dramatic was the increase in the number of immature $(4 \mathrm{x})(P=0.004)$ and partially mature $(4 \mathrm{x})(P=$ 0.001 ) vessels observed after long-term LNG-IUS use (Group II) compared with control group (Fig. 5). The vessel section number of mature vessels $/ \mathrm{mm}$ was significantly decreased after short- or long-term exposure to LNG $(P<0.0001)$ compared with the control group (Fig. 5). Comparison between the long- and short-term LNG groups shows an increased vascularization in short-term LNG users, which is mainly associated with the development of naked vessels. The number of vessel sections of partially mature and mature vessels was not different between the short and long duration of LNG-IUS treatment.

\section{The relative vascular area is increased in endometrium from women treated with levonorgestrel}

The relative vascular area, corresponding to the ratio of vessel surface to the total tissue area, was significantly increased in endometrium of LNG users (Group I and II) when compared with the control group. This significant increase was observed for naked (pericytes-free) vessels scored 0 in the short-term LNG group $(P<0.0001)$ and long-term LNG group $(P=0.0015)$. The relative vascular area of mature vessels decreased in short-term LNG group when compared with the control $(P<0.0001)$ and long-term LNG groups $(P=0.0002)$ (Fig. 6A).

The duration of LNG exposure also dramatically influenced the extent of vascularization and vessel maturation. 
Although $\alpha$ SMA-coated vessels (score 2) were the most prevalent vessels in control group, short-term exposure to LNG-IUS caused a 4-fold increase of naked ( $\alpha$ SMA negative) vessel area. The relative vascular area of these immature vessels became the predominant vessel type, whereas partially mature and mature vesseled areas became less prevalent after 1 month of treatment. This change in the pattern of endometrial vascularization in short-term treatment could promote vessel leakage and rupture and may explain the transient unwanted spotting and bleeding frequently reported after short-term LNG use. After prolonged treatment, the relative vessel area of naked vessels (score 0) decreased in comparison with that in short-term users (Fig. 6A).

Figure 4: Staining of $\alpha \operatorname{SMA}(a, c, e)$ and $M H C(b, d, f)$ on serial sections in Control group $(a, b)$, short-term LNG group $(c, d)$ and long-term LNG group (e,f). (A) Original magnification x200. (B) Ratio of number vessel sections with positive MHC staining on total number of vessel sections with positive $\alpha$ SMA staining in Control group $(n=$ 10), short-term LNG group $(n=8)$ and long-term $L N G$ group $(n=11)$

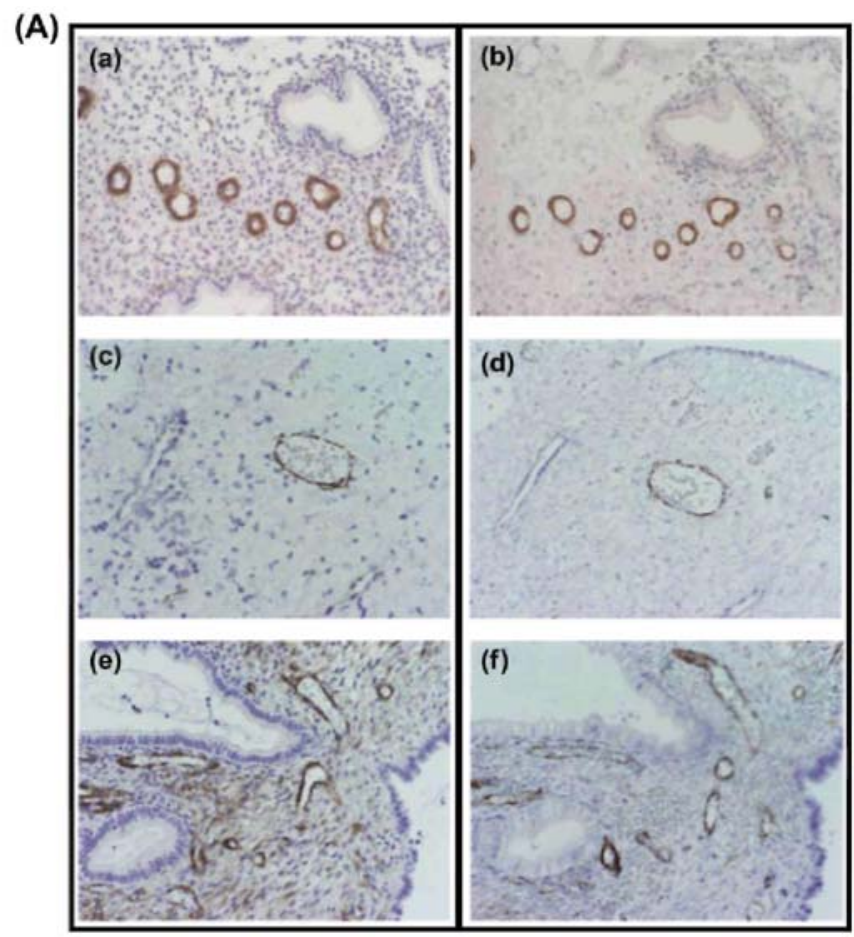

(B)

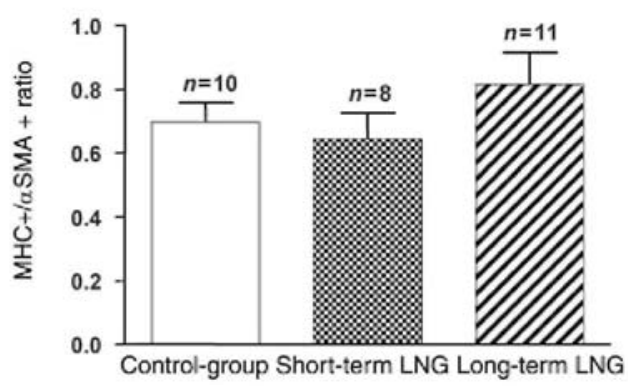

The degree of vessel maturity was different among the three groups. The percentage of the total vascular area occupied by immature vessels (devoid of pericytes) was considerably higher in short-term LNG-IUS users (Group I) than in control group $(P<0.0001)$ (Fig. 6B). No difference was shown in the proportion of total vascular area occupied by partially mature vessels (score 1) between the three groups. A significant decrease was observed in the proportion of mature vessels (score 2) in the short-term LNG group when compared with the control group $(P<0.0001)$ or the long-term LNG group $(P=0.0002)$ (Fig. 6B).

\section{Vessel area varies according to the duration of treatment with LNG-IUS}

The mean vessel surfaces were determined by a computer assisted method for each category of vessels in both LNG groups and the control group (Fig. 7). Immature, partially mature and mature vessels were smaller in short- 
term LNG users when compared with the control group $(P<0.0001)$. In contrast, larger vessels were observed in long-term LNG users, compared with the short-term LNG and control group $(P<0.0001)$. However, the proportion of vessels coated with pericytes increased progressively with time, indicating a trend towards a normalization of vessel structure.

\section{DISCUSSION}

Characteristics of endometrial vessels have not been thoroughly documented in women with short- or long-term exposure to LNG-IUS.

LNG-IUS is an intrauterine contraceptive system, also licensed for the treatment of menorrhagia. The system increases apoptosis of the endometrial glands (Maruo et al., 2001) and promotes decidualization of the stroma (Silverberg et al., 1986). It reduces menstrual blood loss (Andersson and Rybo, 1990; Gupta et al., 2006) and its efficacy in the medical management of dysfunctional uterine bleeding is similar to that of endometrial resection (Gupta et al., 2006). Despite this beneficial effect on menstrual blood loss, compliance is hampered because of the initial troublesome side effect of breakthrough bleeding commonly seen during the first 1-6 months after insertion of the LNG-IUS (Lahteenmaki et al., 1998).

Several factors contribute to BTB and their down- or up-regulation is a determinant for activation and maintenance of bleeding. Progesterone receptor (PR) and estrogen receptors (ER) expression is dramatically reduced in epithelial and stromal cells of LNG-IUS users endometrium (Zhu et al., 1999; Jones and Critchley, 2000; Galant et al., 2004; Vereide et al., 2006). Our immunohistochemical study of PR expression also showed a significant down-regulation in short- and long-term LNG groups compared to a control group (data not shown). Our findings are thus in accordance with the previous studies. The decreased levels of PR may contribute to the contraceptive efficacy of this type of IUS but also to LNG-IUS-induced irregular bleeding. Vessel structure and integrity are indeed altered by progestin use, with changes in the shape, dilatation, the basement membrane components and pericyte support of vessels (Hickey et al., 2000; Hickey and Fraser, 2003). Perivascular cells and, in particular, pericytes provide structural stability and play a key role in blood vessel maturation during angiogenesis (Hanahan, 1997; Kohnen et al., 2000; Carmeliet, 2004). It has been proposed that, in women with BTB, there is an increased endometrial vascular fragility, leading to increased susceptibility to damage and rupture (Hickey et al., 2000). This concept is supported by the finding that pericytes are reduced around microvessels in the endometrium of women using progestin-only contraception who suffer from BTB compared with those without BTB (Rogers et al., 2000).

Changes in vessel density with the associated decrease in the stromal support have also been hypothesized to contribute to vascular fragility and BTB (Hickey et al., 2000; Rogers et al., 2000; Jondet et al., 2005). Finally, a decreased expression of myosin has been documented in arterioles of women suffering from menorrhagia (Abberton et al., 1999; Girling and Rogers, 2005). This decreased vascular smooth muscle cells (VSMCs) differentiation results in a lack of contractile activity and could participate to the pathogenesis of BTB.

Figure 5: Proportion of vessel number $/ \mathrm{mm}^{2}$ in short-term LNG group $(n=8)$ and long-term LNG group $(n=11)$ compared with control group $(n=10)$ Each vessel category was represented as the percentage in each group

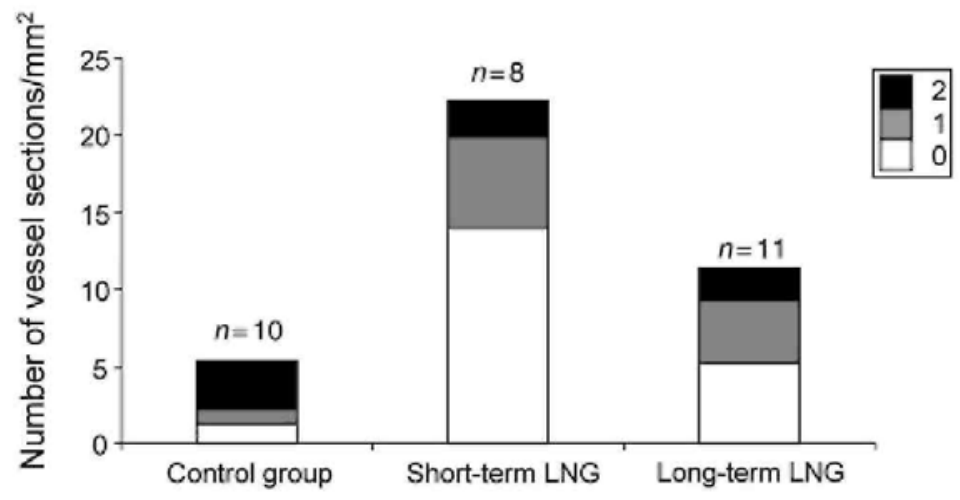


Figure 6: Relative vasculature area and percentage of each vessel category. (A) Relative vasculature area defined as the ratio between the vessel surface to total tissue area, in endometrium, in short-term LNG group $(n=8)$ and long-term $L N G$ group $(n=11)$ compared with control group $(n=10)(* * P<0.001 ; * * * P<0.0001)$. (B) Percentage of each vessel category in control group, short-term LNG group and long-term LNG group.

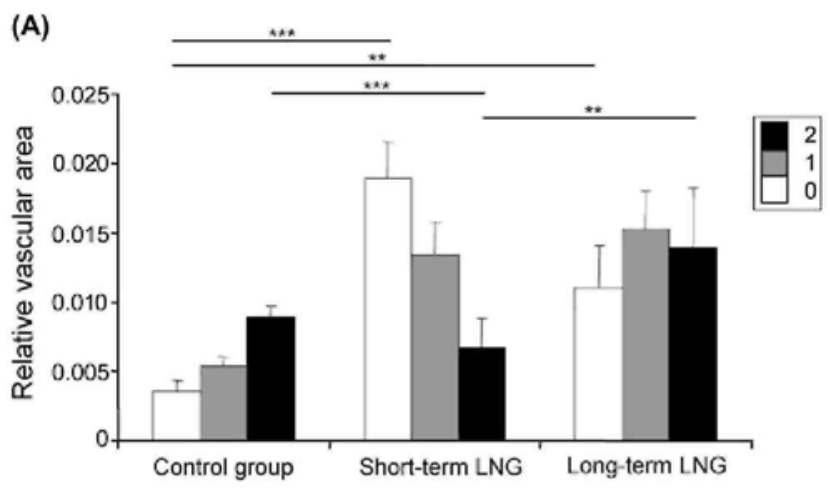

(B)

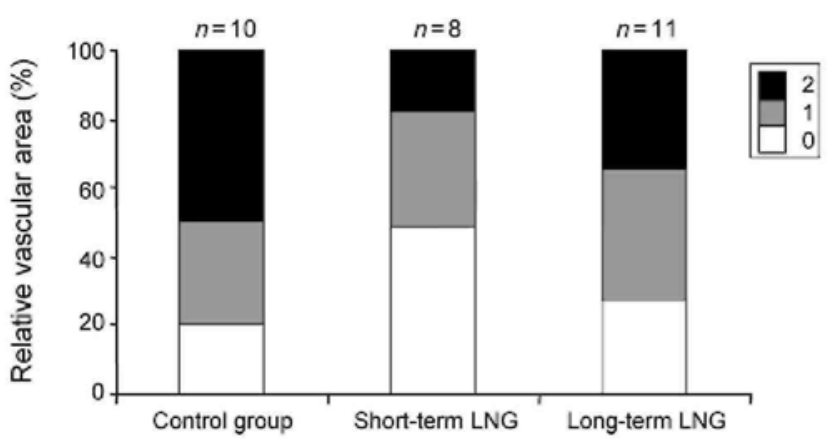

Figure 7: Mean vessel surfaces $\left(\mu \mathrm{m}^{2}\right)$ were determined by computer assisted method for each category of vessels in short-term LNG group $(n=8)$, long-term LNG group $(n=11)$ and the control group $(n=10)(* * * P<0.0001)$

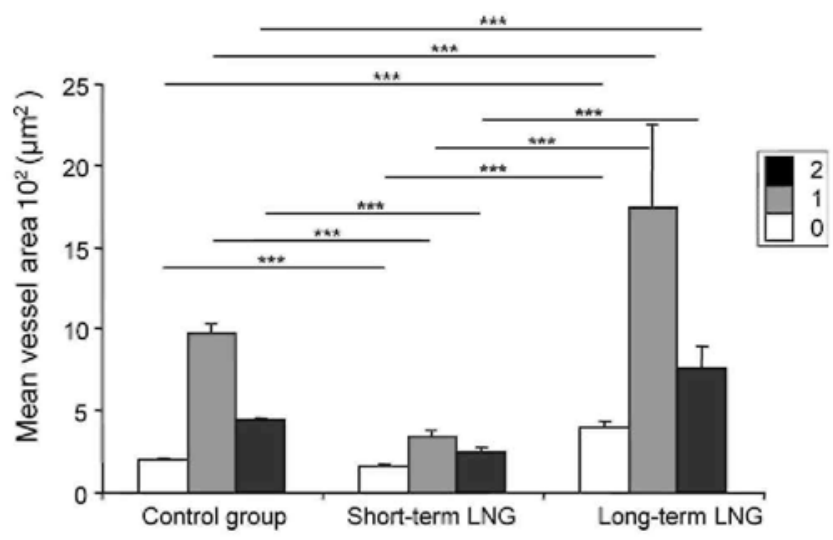

In the present study, we analyzed the vasculature of endometrium under the influence of LNG-IUS for a short or a long period of time. Endometrial angiogenesis in LNG users results indeed in an extensive network of small naked or incompletely $\alpha$ SMA-coated vessels. Short-term LNG-IUS use was associated with a dramatic increase in the number of small naked and partially $\alpha$ SMA-coated vessels $/ \mathrm{mm}^{2}$ (respectively, 11.5 and 6 times). The total tissue area occupied by these vessels was also considerably increased (respectively, 6 and 2.5 times). Altogether, our quantitative data provide a rationale for the excessive bleeding and spotting documented in these patients. Our data are in accordance with the observation of McGavigan et al. (2003) who have shown an increase in blood vessel density after a short-term exposure to LNG-IUS (McGavigan et al., 2003).

In this study, long-term LNG use was associated with a significant decrease in the number of naked vessels in comparison with short-term LNG use. The relative vascular area of immature naked vessels was also reduced, 
whereas that of mature vessels was increased in comparison with short-term users. Despite this progressive trend towards normalization of vessel structure, all vessels became larger after long-term use. This may also contribute to explain the unwanted BTB that may persist in some women, even after prolonged exposure.

Hague et al. (2002) also previously showed an enhanced vascular density in long-term LNG users (Hague et al., 2002), but did not discriminate between vessel types. Other studies have demonstrated changes similar to those described in our study in endometrium of women subcutaneously implanted with Norplant (Rogers et al., 1993; Hickey et al., 1999). An increase in total endometrial vessel density and an increase of negative $\alpha$ SMA vessels were described by Rogers et al. $(1993 ; 2000)$. These congruent data strongly suggest that the vascular changes observed in our analysis can be attributed to the progestin present in the LNG-IUS rather than to an IUD effect. Indeed, copper IUDs classically do not elicit the histological changes described here and in Norplant users (Sheppard, 1987).

Jondet et al. (2005) found a reduction of microvessel number with a concomitant dilatation in LNG-IUS users. They failed to discriminate maturation between vessel types which appears in this study to be a key parameter to attempt to correlate blood vessel changes with clinical features of bleeding. Angiogenesis in the endometrium involves the development of arterioles and elaboration of a capillary network. These events of maturation of arterioles involve a regulated VSMC differentiation, i.e. reduced by progestin. In endometrium of menorrhagic women, MHC expression was found to be reduced in VSMC of spiral arterioles. (Abberton et al., 1999). In this study, in each group, $\sim 70 \%$ of all $\alpha$ SMA positive vessels also expressed the contractile myosin suggesting that changes in the contractile phenotypes are not responsible for the increased bleeding of these women.

Altogether, our results show that LNG exposition leads to (i) a rapid increase of the number and area of sections of fragile naked vessels, (ii) the formation of smaller vessels after 1-3 months of treatment for all vessel categories and (iii) the enlargement of naked, partially mature and mature vessels after long-time exposure. These data support the clinical use of strategies that may minimize vascular morphology changes. Recently, Critchley et al. (2006) proposed an intermittent antiprogestin treatment during the first 3 months coincident with the highest bleeding and vascular fragility. Future approaches should be considered to limit these effects of progestogen on the endometrial blood vessel remodelling.

\section{Acknowledgements}

We thank Professor Agnès Noël for scientific advice. We also thank Isabelle Dasoul, Patricia Gavitelli and Emilie Feyereisen for their excellent technical assistance. This work was supported by grants from the Communauté française de Belgique (Actions de Recherches Concertées), the Commission of European Communities, NOE no LSHM-CT-2004-512040 'EMBIC', the Fonds de la Recherche Scientifique Médicale, the Fonds National de la Recherche Scientifique (F.N.R.S., Belgium), the Fonds spéciaux de la Recherche (University of Liège), the Fondation Léon Fredericq (University of Liège), the D.G.T.R.E. from the 'Région Wallonne', the F.S.E. (Fonds Social Européen), the Fonds d'Investissements de la Recherche Scientifique (F.I.R.S., CHU, Liège, Belgium) and the Interuniversity Attraction Poles Programme, Belgian Science Policy (Brussels, Belgium).

\section{References}

Abberton KM, Healy DL, Rogers PA. Smooth muscle alpha actin and myosin heavy chain expression in the vascular smooth muscle cells surrounding human endometrial arterioles. Hum Reprod 1999;14:3095-3100.

Andersson JK, Rybo G. Levonorgestrel-releasing intrauterine device in the treatment of menorrhagia. $\mathrm{Br} J$ Obstet Gynaecol 1990;97:690-694.

Carmeliet P. Manipulating angiogenesis in medicine. J Intern Med 2004;255:538-561.

Chegini N, Rhoton-Vlasak A, Williams RS. Expression of matrix metalloproteinase-26 and tissue inhibitor of matrix metalloproteinase- 3 and -4 in endometrium throughout the normal menstrual cycle and alteration in users of levonorgestrel implants who experience irregular uterine bleeding. Fertil Steril 2003;80:564-570.

Critchley HO, Wang H, Jones RL, Kelly RW, Drudy TA, Gebbie AE, Buckley CH, McNeilly AS, Glasier AF. Morphological and functional features of endometrial decidualization following long-term intrauterine 
levonorgestrel delivery. Hum Reprod 1998;13:1218-1224.

Critchley HO, Kelly RW, Baird DT, Brenner RM. Regulation of human endometrial function: mechanisms relevant to uterine bleeding. Reprod Biol Endocrinol 2006;4(Suppl 1):S5.

Galant C, Vekemans M, Lemoine P, Kokorine I, Twagirayezu P, Henriet P, Picquet C, Rigot V, Eeckhout Y, Courtoy PJ et al. Temporal and spatial association of matrix metalloproteinases with focal endometrial breakdown and bleeding upon progestin-only contraception. J Clin Endocrinol Metab 2000;85:4827-4834.

Galant C, Berliere M, Dubois D, Verougstraete JC, Charles A, Lemoine P, Kokorine I, Eeckhout Y, Courtoy PJ, Marbaix E. Focal expression and final activity of matrix metalloproteinases may explain irregular dysfunctional endometrial bleeding. Am J Pathol 2004;165:83-94.

Girling JE, Rogers PA. Recent advances in endometrial angiogenesis research. Angiogenesis 2005;8:89-99.

Gordon H, Sweets HH, Jr. A simple method for the silver impregnation of reticulum. Am J Pathol. 1936;12:545551.

Gupta B, Mittal S, Misra R, Deka D, Dadhwal V. Levonorgestrel-releasing intrauterine system vs. transcervical endometrial resection for dysfunctional uterine bleeding. Int J Gynaecol Obstet 2006;95:261-266.

Hague S, MacKenzie IZ, Bicknell R, Rees MC. In-vivo angiogenesis and progestogens. Hum Reprod 2002;17:786-793.

Hanahan D. Signaling vascular morphogenesis and maintenance. Science 1997;277:48-50.

Hickey M, Fraser I. Human uterine vascular structures in normal and diseased states. Microsc Res Tech 2003;60:377-389.

Hickey M, Simbar M, Markham R, Young L, Manconi F, Russell P, Fraser IS. Changes in vascular basement membrane in the endometrium of Norplant users. Hum Reprod 1999;14:716-721.

Hickey M, Dwarte D, Fraser IS. Superficial endometrial vascular fragility in Norplant users and in women with ovulatory dysfunctional uterine bleeding. Hum Reprod 2000;15:1509-1514.

Jondet M, Letellier B, Verdys MT. Endometrial vascularization in levonorgestrel intrauterine device users; computerized microvessel measurement study. Contraception 2005;71:60-64.

Jones RL, Critchley HO. Morphological and functional changes in human endometrium following intrauterine levonorgestrel delivery. Hum Reprod 2000;15(Supp13):162-172.

Kohnen G, Campbell S, Jeffers MD, Cameron IT. Spatially regulated differentiation of endometrial vascular smooth muscle cells. Hum Reprod 2000;15:284-292.

Kokorine I, Marbaix E, Henriet P, Okada Y, Donnez J, Eeckhout Y, Courtoy PJ. Focal cellular origin and regulation of interstitial collagenase (matrix metalloproteinase-1) are related to menstrual breakdown in the human endometrium. J Cell Sci 1996;109:2151-2160.

Lahteenmaki P, Haukkamaa M, Puolakka J, Riikonen U, Sainio S, Suvisaari J, Nilsson CG. Open randomised study of use of levonorgestrel releasing intrauterine system as alternative to hysterectomy. BMJ 1998;316:11221126.

Maruo T, Laoag-Fernandez JB, Pakarinen P, Murakoshi H, Spitz IM, Johansson E. Effects of the levonorgestrelreleasing intrauterine system on proliferation and apoptosis in the endometrium. Hum Reprod 2001;16:21032108 .

McGavigan CJ, Dockery P, Metaxa-Mariatou V, Campbell D, Stewart CJ, Cameron IT, Campbell S. Hormonally mediated disturbance of angiogenesis in the human endometrium after exposure to intrauterine levonorgestrel. Hum Reprod 2003;18:77-84.

Noyes RW, Hertig AT, Rock J. Dating the endometrial biopsy. Fertil Steril 1950;1:3-25. 
Rogers PA, Abberton KM. Endometrial arteriogenesis: vascular smooth muscle cell proliferation and differentiation during the menstrual cycle and changes associated with endometrial bleeding disorders. Microsc Res Tech 2003;60:412-419.

Rogers PA, Au CL, Affandi B. Endometrial microvascular density during the normal menstrual cycle and following exposure to long-term levonorgestrel. Hum Reprod 1993;8:1396-1404.

Rogers PA, Plunkett D, Affandi B. Perivascular smooth muscle alpha-actin is reduced in the endometrium of women with progestin-only contraceptive breakthrough bleeding. Hum Reprod 2000;15(Suppl 3):78-84.

Runic R, Schatz F, Wan L, Demopoulos R, Krikun G, Lockwood CJ. Effects of Norplant on endometrial tissue factor expression and blood vessel structure. J Clin Endocrinol Metab 2000;85:3853-3859.

Russ JC. Image measurments. The image processing Handbook, Florida, 1999a, pp. 509-574.

Russ JC. Processing binary images. The Image Processing Handbook, 3rd edn., Florida, 1999b, pp. 431-508.

Sheppard BL. Endometrial morphological changes in IUD users: a review. Contraception 1987;36:1-10.

Silverberg SG, Haukkamaa M, Arko H, Nilsson CG, Luukkainen T. Endometrial morphology during long-term use of levonorgestrel-releasing intrauterine devices. Int J Gynecol Pathol 1986;5:235-241.

Skinner JL, Riley SC, Gebbie AE, Glasier AF, Critchley HO. Regulation of matrix metalloproteinase-9 in endometrium during the menstrual cycle and following administration of intrauterine levonorgestrel. Hum Reprod 1999;14:793-799.

Vekemans M. Use of long-acting contraceptives. Lancet 1995;346:1165. Vekemans M, Delvigne A, Paesmans M. Continuation rates with a levonorgestrel-releasing contraceptive implant (Norplant). A prospective study in Belgium. Contraception 1997;56:291-299.

Vereide AB, Kaino T, Sager G, Arnes M, Orbo A. Effect of levonorgestrel IUD and oral medroxyprogesterone acetate on glandular and stromal progesterone receptors (PRA and PRB), and estrogen receptors (ER-alpha and ER-beta) in human endometrial hyperplasia. Gynecol Oncol 2006;101:214-223.

Vincent AJ, Malakooti N, Zhang J, Rogers PA, Affandi B, Salamonsen LA. Endometrial breakdown in women using Norplant is associated with migratory cells expressing matrix metalloproteinase-9 (gelatinase B). Hum Reprod 1999;14:807-815.

Vincent AJ, Zhang J, Ostor A, Rogers PA, Affandi B, Kovacs G, Salamonsen LA. Decreased tissue inhibitor of metalloproteinase in the endometrium of women using depot medroxyprogesterone acetate: a role for altered endometrial matrix metalloproteinase/tissue inhibitor of metalloproteinase balance in the pathogenesis of abnormal uterine bleeding? Hum Reprod 2002;17:1189-1198.

Zhu P, Liu X, Luo H, Gu Z, Cheng J, Xu R, Lian S, Wu S, Wang J. The effect of a levonorgestrel-releasing intrauterine device on human endometrial oestrogen and progesterone receptors after one year of use. Hum Reprod 1999;14:970-975. 